\title{
DYNAMICS OF A DISCRETE ECO-EPIDEMIOLOGICAL MODEL WITH DISEASE IN THE PREY
}

\author{
LOPO F. DE JESUS, CÉSAR M. SILVA, AND HELDER VILARINHO
}

\begin{abstract}
Using Mickens nonstandard method, we obtain a discrete family of nonautonomous eco-epidemiological models that include general functions corresponding to the predation of the infected and uninfected preys. We obtain results on the persistence and extinction of the infected preys assuming that the bi-dimensional predator-prey subsystem that describes the dynamics in the absence of the infection satisfies some assumptions. Some examples and simulations are undertaken to illustrate our results.
\end{abstract}

\section{INTRODUCTION}

In many situations eco-epidemiological models describe more accurately some ecological system than classical Lotka-Volterra models where the disease is not taken into account. It is known that the inclusion of infected classes in predatorprey models substantially change the dynamics of the original model. In particular, the inclusion of infected classes in the model can have a considerable impact on the population size of the predator-prey community [6, 8].

Lately, several works related to eco-epidemiological models have appeared in the literature. In 2, the authors study the extinction and persistence of the disease in some eco-epidemiological systems; in 1] the global stability of a delayed ecoepidemiological model with Holling type III functional response is addressed, and in 14 the authors study an eco-epidemiological model with harvesting.

We note that the parameters in the eco-epidemiological models referred above are constant. On the other hand, to make models more consistent with reality it is seldom important to consider parameters that vary in time. Recently, several eco-epidemiological models with time varying parameters, particularly models with periodic coefficients have been studied [3, 4, 8, 9, 20, 10]. In the more general situation of nonautonomous models that are not necessarily periodic, threshold conditions for the extinction and persistence of the infected preys are obtained in 20. for a family of non-autonomous eco-epidemiological models with disease in the prey and no predation on uninfected preys. The results in that paper are generalised in 11 for a class of non-autonomous eco-epidemiological models that include general functions corresponding to the predation on uninfected prey and also to the vital dynamics of uninfected prey and predator populations. Note that already in 21] a family of models that include predation on uninfected preys

Date: July 21, 2020.

2010 Mathematics Subject Classification. 92D30, 39A60.

Key words and phrases. Eco-epidemiological model, difference equations, persistence, extinction.

L. de Jesus, C. M. Silva and H. Vilarinho were partially supported by FCT through CMA-UBI (project UIDB/MAT/00212/2020). 
where considered, assuming that predation on uninfected prey is given by a bilinear functional response and also some particular form for the vital dynamics associated with uninfected preys and predators.

The approach in [11] is very different from the one in 20] and 21]: in [11] the uninfected subsystem corresponding to the dynamics of preys and predators in the absence of disease is not assumed to follow some special law but instead the hypothesis are on the stability of the referred uninfected subsystem. This approach allows the application of the results in [11] to eco-epidemiological models constructed from previously studied predator-prey model that satisfies the stability assumptions made.

In all the previous situations the models involved are continuous. In contrast, in this paper we consider a discrete version of the model in [11] obtained by applying Mickens discretization method. For the obtained model we derive a discrete version of the main result in that paper regarding the threshold dynamics of the model. We note that in [7] a discrete eco-epidemiological model was already studied. In contrast with our nonautonomous model, in that paper the model considered is autonomous and assumes no predation on uninfected preys. Additionally, in that paper the discretization method is very different from ours, resulting in a very different form for the equations obtained.

The structure of the present work is the following: in section 2 we derive our model from the corresponding continuous model using Mickens nonstandard discretization scheme, establish our setting and some preliminary results; in section 3 we obtain our main result on extinction and persistence of the infective prey; finally, in section 4, we consider some particular models that illustrate our results.

\section{A GENERAL ECO-EPIDEMIOLOGICAL MODEL WITH DISEASE IN THE PREY}

We consider the following non-autonomous eco-epidemiological model:

$$
\left\{\begin{array}{l}
S^{\prime}=\Lambda(t)-\mu(t) S-a(t) f(S, I, P) P-\beta(t) S I \\
I^{\prime}=\beta(t) S I-\eta(t) g(S, I, P) I-c(t) I \\
P^{\prime}=(r(t)-b(t) P) P+\gamma(t) a(t) f(S, I, P) P+\theta(t) \eta(t) g(S, I, P) I
\end{array}\right.
$$

where $S, I$ and $P$ correspond, respectively, to the susceptible prey, infected prey and predator, $\Lambda(t)$ is the birth rate, $\mu(t)$ is the death rate of susceptible preys, $\beta(t)$ is the incidence rate of the disease, $\eta(t)$ is the predation rate of infected prey, $c(t)$ is the death rate in the infective class, $\gamma(t)$ is the rate converting susceptible prey into predator (biomass transfer), $\theta(t)$ is the rate of converting infected prey into predator, $r(t)-b(t) P$ represent the vital dynamics of the predator populations, $a(t) f(S, I, P)$ is the predation of susceptible prey and $\eta(t) g(S, I, P)$ is the predation of infected prey. It is assumed that only susceptible preys $S$ are capable of reproducing, i.e, the infected prey is removed by death (including natural and disease-related death) or by predation before having the possibility of reproducing.

The aim of this work is to discuss the uniform strong persistence and extinction of the infectives $I$ of the discrete counterpart of the system (1). A possible discretization of the above model, with stepsize $h$, derived by applying Mickens' 
nonstandard finite difference method [13, leads to the following set of equations:

$$
\left\{\begin{aligned}
S(n h+h)-S(n h)= & h \Lambda(n h)-h \mu(n h) S(n h+h) \\
& -h a(n h) f(S(n h+h), I(n h), P(n h)) P(n h) \\
& -h \beta(n h) S(n h+h) I(n h) \\
I(n h+h)-I(n h)= & h \beta(n h) S(n h+h) I(n h) \\
& -h \eta(n h) g(S(n h), I(n h), P(n h)) I(n h+h) \\
& -h c(n h) I(n h+h) \\
P(n h+h)-P(n h)= & h(r(n h)-b(n h) P(n h+h)) P(n h)+h \gamma(n h) a(n h) \times \\
& \times f(S(n h+h), I(n h), P(n h)) P(n h) \\
& +h \theta(n h) \eta(n h) g(S(n h), I(n h), P(n h)) I(n h+h)
\end{aligned}\right.
$$

Using the notation $\xi_{n}=h \xi(n h)$ for $g=\Lambda, \mu, a, \beta, \eta, c, r, b$ and also $\zeta_{n}=\zeta(n h)$ for $\zeta=\gamma, \theta$, we obtain the following system of difference equations:

$$
\left\{\begin{array}{l}
S_{n+1}-S_{n}=\Lambda_{n}-\mu_{n} S_{n+1}-a_{n} f\left(S_{n+1}, I_{n}, P_{n}\right) P_{n}-\beta_{n} S_{n+1} I_{n} \\
I_{n+1}-I_{n}=\beta_{n} S_{n+1} I_{n}-\eta_{n} g\left(S_{n}, I_{n}, P_{n}\right) I_{n+1}-c_{n} I_{n+1} \\
P_{n+1}-P_{n}=\left(r_{n}-b_{n} P_{n+1}\right) P_{n}+\gamma_{n} a_{n} f\left(S_{n+1}, I_{n}, P_{n}\right) P_{n}+\theta_{n} \eta_{n} g\left(S_{n}, I_{n}, P_{n}\right) I_{n+1}
\end{array} .\right.
$$

We will assume that

H1) $\left(\Lambda_{n}\right),\left(a_{n}\right),\left(\beta_{n}\right),\left(\eta_{n}\right),\left(c_{n}\right),\left(r_{n}\right),\left(b_{n}\right),\left(\gamma_{n}\right)$ and $\left(\theta_{n}\right)$ are bounded and nonnegative sequences and $0<\mu_{n} \leqslant c_{n}$;

H2) $\left(\Lambda_{n}\right),\left(r_{n}\right)$ and $\left(b_{n}\right)$ are bounded away from zero;

H3) $f, g:\left(\mathbb{R}_{0}^{+}\right)^{3} \rightarrow \mathbb{R}$ are $C^{1}$ nonnegative; for fixed $x, z \geqslant 0, y \mapsto f(x, y, z)$ and $y \mapsto g(x, y, z)$ are nonincreasing; for fixed $y, z \geqslant 0, x \mapsto f(x, y, z)$ is nondecreasing and $x \mapsto g(x, y, z)$ is nonincreasing; for fixed $x, y \geqslant 0, z \mapsto$ $g(x, y, z)$ is nonincreasing and $z \mapsto g(x, y, z)$ is nondecreasing;

H4) there is $\omega \in \mathbb{N}$ such that

$$
\limsup _{n \rightarrow+\infty} \prod_{k=n}^{n+\omega} \frac{1}{1+\mu_{k}}<1
$$

It follows from H(4) that there are constants $K>0$ and $\theta \in] 0,1[$ such that

$$
\prod_{k=m}^{n-1} \frac{1}{1+\mu_{k}}<K \theta^{n-m}
$$

for any $m, n \in \mathbb{N}_{0}$ with $n>m$.

H5) Given $p \in \mathbb{N}$ there is a unique solution $\left(\left(S_{n}, I_{n}, P_{n}\right)\right)_{n \geqslant p}$ of system (2) with initial condition $\left(S_{p}, I_{p}, P_{p}\right) \in\left(\mathbb{R}_{0}^{+}\right)^{3}$.

H6) Any solution of system (2) with nonnegative (resp. positive) initial condition, $\left(S_{q}, I_{q}, P_{q}\right)$ is nonnegative (resp. positive) for all $n \geqslant q$. 
Note that when $f\left(S_{n+1}, I_{n}, P_{n}\right)=S_{n+1}$ and $g\left(S_{n}, I_{n}, P_{n}\right)=P_{n}$ in (2), the equation can be rewritten in explicit form:

$$
\left\{\begin{array}{l}
S_{n+1}=\frac{\Theta_{n}}{\Psi_{n}} \\
I_{n+1}=\frac{\beta_{n} \Theta_{n}+\Psi_{n}}{\Psi_{n} \Phi_{n}} I_{n} \\
P_{n+1}=\frac{\left(1+r_{n}\right) \Psi_{n} \Phi_{n}+\gamma_{n} a_{n} \Theta_{n} \Phi_{n}+\theta_{n} \eta_{n}\left(\Psi_{n}+\beta_{n} \Theta_{n}\right) I_{n}}{\Psi_{n} \Phi_{n}\left(1+b_{n} P_{n}\right)} P_{n}
\end{array}\right.
$$

where $\Psi_{n}=1+\mu_{n}+\beta_{n} I_{n}+a_{n} P_{n}, \Phi_{n}=1+\eta_{n} P_{n}+c_{n}$ and $\Theta_{n}=\Lambda_{n}+S_{n}$. From (4), we conclude that when $f\left(S_{n+1}, I_{n}, P_{n}\right)=S_{n+1}$ system (2) is well defined and H(6) holds. Let us introduce the notation $f^{\ell}=\inf f_{n}$ and $f^{u}=\sup f_{n}$.

To proceed, we need to consider two auxiliary equations. The first one corresponds to the dynamics of preys in the absence of infected preys and predators:

$$
s_{n+1}-s_{n}=\Lambda_{n}-\mu_{n} s_{n+1} .
$$

Rearranging terms, the equation above becomes:

$$
s_{n+1}=\frac{\Lambda_{n}}{1+\mu_{n}}+\frac{s_{n}}{1+\mu_{n}} .
$$

We have the following lemma that was essentially proved in [12]:

Lemma 1. We have the following:

i) The solution of equation (5) with $s_{p}=0$ is the identically null sequence;

ii) All solutions $\left(s_{n}\right)$ of equation (5) with initial condition $s_{0}>0$ are positive for all $n \in \mathbb{N}$

iii) Given a solution $\left(s_{n}\right)$ of equation (5) with initial condition $s_{0} \in\left[\Lambda^{\ell} / \mu^{u}, \Lambda^{u} / \mu^{\ell}\right]$ we have

$$
\frac{\Lambda^{\ell}}{\mu^{u}} \leqslant s_{n} \leqslant \frac{\Lambda^{u}}{\mu^{\ell}}
$$

for all $n \in \mathbb{N}$;

iv) Each fixed solution $\left(s_{n}\right)$ of (5) with initial condition $s_{0} \geqslant 0$ is bounded and globally uniformly attractive on $[0,+\infty)$;

v) There is a constant $D>0$ such that if $\left(s_{n}\right)$ is a solution of (5) and $\left(\tilde{s}_{n}\right)$ is a solution of the system

$$
s_{n+1}=\frac{\Lambda_{n}+s_{n}+\varphi_{n}}{1+\mu_{n}}
$$

with $\tilde{s}_{0}=s_{0}$ then

$$
\sup _{n \geqslant 0}\left|\tilde{s}_{n}-s_{n}\right| \leqslant D \sup _{n \geqslant 0}\left|\varphi_{n}\right| .
$$

vi) There is a constant $E>0$ such that if $\left(s_{n}\right)$ is a solution of (5) and $\left(\tilde{s}_{n}\right)$ is a solution of the system

$$
s_{n+1}=\frac{\Lambda_{n}+s_{n}}{1+\mu_{n}+\psi_{n}}
$$

with $\tilde{s}_{0}=s_{0}$ then there is $N_{1}$ sufficiently large such that

$$
\sup _{n \geqslant N_{1}}\left|\tilde{s}_{n}-s_{n}\right| \leqslant E \sup _{n \geqslant N_{1}}\left|\psi_{n}\right| \text {. }
$$


Proof. Properties (i) to $\mathbf{\nabla}$ ) follow from Lemma 1 in [12. To prove vil), let $\left(s_{n}\right)$ be a solution of (5) and $\left(\tilde{s}_{n}\right)$ be a solution of (7) with $\tilde{s}_{0}=s_{0}$. By (5) and (7), we have

$$
\left(\tilde{s}_{n+1}-s_{n+1}\right)\left(1+\mu_{n}\right)=\tilde{s}_{n}-s_{n}-g_{n} \tilde{s}_{n+1}
$$

Therefore, letting $w_{n}=\left|\tilde{s}_{n}-s_{n}\right|$, we have

$$
w_{n+1}\left(1+\mu_{n}\right) \leqslant w_{n}+\left|g_{n}\right| \tilde{s}_{n+1}
$$

and thus

$$
w_{n+1} \leqslant \frac{w_{n}}{1+\mu_{n}}+\frac{\left|\psi_{n}\right| \tilde{s}_{n+1}}{1+\mu_{n}}
$$

Fix $\varepsilon>0$. By (iii) and iv we get, for $n$ sufficiently large, say $n \geqslant N_{1}$,

$$
w_{n+1} \leqslant \frac{w_{n}}{1+\mu_{n}}+\frac{\left|\psi_{n}\right|}{1+\mu_{n}}\left[\frac{\Lambda^{u}}{\mu^{\ell}+\psi^{\ell}}+\varepsilon\right]
$$

and thus, for $n \geqslant N_{1}$,

$$
\begin{aligned}
w_{n} & \leqslant\left[\frac{\Lambda^{u}}{\mu^{\ell}+\psi^{\ell}}+\varepsilon\right] \sum_{m=0}^{n-1}\left|\psi_{m}\right|\left(\prod_{k=m}^{n-1} \frac{1}{1+\mu_{k}}\right) \\
& \leqslant\left[\frac{\Lambda^{u}}{\mu^{\ell}+\psi^{\ell}}+\varepsilon\right] \sup _{n \geqslant 0}\left|\psi_{n}\right| K \sum_{m=0}^{n-1} \theta^{n-m} \\
& \leqslant\left[\frac{\Lambda^{u}}{\mu^{\ell}+\psi^{\ell}}+\varepsilon\right] \frac{K \theta}{1-\theta} \sup _{n \geqslant 0}\left|\psi_{n}\right| .
\end{aligned}
$$

Defining $E=K \theta\left[\Lambda^{u} /\left(\mu^{\ell}+\psi^{\ell}\right)+\varepsilon\right] /(1-\theta)$, we get

$$
\sup _{n \geqslant N_{1}}\left|\tilde{s}_{n}-s_{n}\right|=\sup _{n \geqslant N_{1}} w_{n} \leqslant w_{n} \leqslant E \sup _{n \geqslant N_{1}}\left|\psi_{n}\right|,
$$

and the result follows.

We also need to consider the equation:

$$
y_{n+1}-y_{n}=\left(r_{n}-b_{n} y_{n+1}\right) y_{n} .
$$

Rearranging terms, we get:

$$
y_{n+1}=\frac{r_{n} y_{n}+y_{n}}{1+b_{n} y_{n}}
$$

The following lemma holds.

Lemma 2. We have the following:

i) The solution of equation (8) with $y_{p}=0$ is the identically null sequence;

ii) All solutions $\left(y_{n}\right)$ of equation (8) with initial condition $y_{0}>0$ are positive for all $n \in \mathbb{N}$

iii) Given a solution $\left(y_{n}\right)$ of equation (8) with initial condition $y_{0} \in\left[r^{\ell} / b^{u}, r^{u} / b^{\ell}\right]$ we have

for all $n \in \mathbb{N}$;

$$
\frac{r^{\ell}}{b^{u}} \leqslant y_{n} \leqslant \frac{r^{u}}{b^{\ell}}
$$

iv) Each fixed solution $\left(y_{n}\right)$ of (8) with initial condition $y_{0}>0$ is bounded and globally uniformly attractive on $] 0,+\infty)$; 
v) There is a constant $E>0$ such that, if $\left(y_{n}\right)$ is a solution of (8) and $\left(\tilde{y}_{n}\right)$ is a solution of the system

$$
y_{n+1}=\frac{r_{n} y_{n}+y_{n}}{1+\left(b_{n}+g_{n}\right) y_{n}}, \quad n=0,1, \ldots
$$

with $\tilde{y}_{0}=y_{0}$ then there is $N_{1}$ sufficiently large such that

$$
\sup _{n \geqslant N_{1}}\left|\tilde{y}_{n}-y_{n}\right| \leqslant E \sup _{n \geqslant N_{1}}\left|g_{n}\right| .
$$

vi) There is a constant $G>0$ such that, if $\left(y_{n}\right)$ is a solution of (8) and $\left(\tilde{y}_{n}\right)$ is a solution of the system

$$
y_{n+1}=\frac{\left(r_{n}+h_{n}\right) y_{n}+y_{n}}{1+b_{n} y_{n}}, \quad n=0,1, \ldots
$$

with $\tilde{y}_{0}=y_{0}$ then there is $N_{2}$ sufficiently large such that

$$
\sup _{n \geqslant N_{2}}\left|\tilde{y}_{n}-y_{n}\right| \leqslant G \sup _{n \geqslant N_{2}}\left|h_{n}\right| .
$$

Proof. With the change of variable $w_{n}=1 / y_{n}$, equation (8) becomes

$$
w_{n+1}=\frac{b_{n}}{r_{n}+1}+\frac{w_{n}}{r_{n}+1}
$$

equation (9) becomes

$$
w_{n+1}=\frac{w_{n}+b_{n}+g_{n}}{r_{n}+1} .
$$

and equation (10) becomes

$$
w_{n+1}=\frac{w_{n}+b_{n}}{1+r_{n}+h_{n}} .
$$

Using Lemma 1, we obtain (iii) to vii). Property (i) is immediate.

We must assume the following:

H7) Each solution of (2) with positive initial condition is bounded and there is a bounded region $\mathcal{R}$ that contains the $\omega$-limit of all solutions of (2) with positive initial conditions.

Notice in particular that condition H(7) implies that there is $L>0$ such that, for each solution $\left(S_{n}, I_{n}, P_{n}\right)$ we have

$$
\limsup _{t \rightarrow+\infty}\left(S_{n}+I_{n}+P_{n}\right)<L .
$$

The next lemma shows that, when $g(S, I, P)=g_{0}(S, I) P$, there is an invariant region that attracts all orbits of system (2).

Lemma 3. Assume that $g(S, I, P)=g_{0}(S, I) P$. Then, there is $L>0$ such that, for any solution $\left(S_{n}, I_{n}, P_{n}\right)$ of (2), with nonnegative initial conditions, there is $T \in \mathbb{N}$ such that

$$
S_{n}+I_{n}+P_{n} \leqslant L \text { for } n \geqslant T .
$$


Proof. Let $\left(S_{n}, I_{n}, P_{n}\right)$ be a solution of (2) with nonnegative initial conditions $S_{q}=$ $s_{q}, I_{q}=i_{q}$ and $P_{q}=p_{q}$. Adding the first two equations in (2) and writing $N_{n}=$ $S_{n}+I_{n}$, we get

$$
\begin{aligned}
N_{n+1}-N_{n}= & \Lambda_{n}-\mu_{n} S_{n+1}-c_{n} I_{n+1}-a_{n} f\left(S_{n+1}, I_{n}, P_{n}\right) P_{n} \\
& -\eta_{n} g_{0}\left(S_{n+1}, I_{n}\right) P_{n} I_{n+1} \\
\leqslant & \Lambda_{n}-\mu_{n} N_{n+1},
\end{aligned}
$$

since $\mu_{n}=\min \left\{\mu_{n}, c_{n}\right\}$. Thus

$$
N_{n+1} \leqslant \frac{\Lambda_{n}}{1+\mu_{n}}+\frac{N_{n}}{1+\mu_{n}} .
$$

By (iii) and (iv) in Lemma 11 we conclude that, for any given $\varepsilon>0$, we have $S_{n}+I_{n}=N_{n} \leqslant s_{n} \leqslant \Lambda^{u} / \mu^{\ell}+\varepsilon$, where $s_{n}$ is a solution of (5) with initial condition $s_{q}=N_{q}$, for $n$ sufficiently large, say $n \geqslant N_{1}$.

By the third equation in (2) we obtain

$$
\begin{aligned}
P_{n+1} & =\frac{P_{n}+r_{n} P_{n}+\gamma_{n} a_{n} f\left(S_{n+1}, I_{n}, P_{n}\right) P_{n}+\theta_{n} \eta_{n} g_{0}\left(S_{n+1}, I_{n}\right) P_{n} I_{n+1}}{1+b_{n} P_{n}} \\
& \leqslant \frac{\left[r_{n}+\gamma_{n} a_{n} f\left(\Lambda^{u} / \mu^{\ell}+\varepsilon, 0,0\right)+\theta_{n} \eta_{n} g_{0}\left(0, \Lambda^{u} / \mu^{\ell}+\varepsilon\right)\left(\Lambda^{u} / \mu^{\ell}+\varepsilon\right)\right] P_{n}+P_{n}}{1+b_{n} P_{n}}
\end{aligned}
$$

for $n \geqslant N_{1}$. By (iii) and [iv) in Lemma 2, we conclude that, for any given $\delta>0$, there is $N_{2} \geqslant N_{1}$ such that, for all $n \geqslant N_{2}$

$$
\begin{aligned}
P_{n} & \leqslant \frac{\sup _{n \geqslant q}\left\{r_{n}+\gamma_{n} a_{n} f\left(\Lambda^{u} / \mu^{\ell}+\varepsilon, 0,0\right)+\theta_{n} \eta_{n} g_{0}\left(0, \Lambda^{u} / \mu^{\ell}+\varepsilon\right)\left(\Lambda^{u} / \mu^{\ell}+\varepsilon\right)\right\}}{b^{\ell}}+\delta \\
& \leqslant \frac{r^{u}+\gamma^{u} a^{u} f\left(\Lambda^{u} / \mu^{\ell}+\varepsilon, 0,0\right)+\theta^{u} \eta^{u} g_{0}\left(0, \Lambda^{u} / \mu^{\ell}+\varepsilon\right)\left(\Lambda^{u} / \mu^{\ell}+\varepsilon\right)}{b^{\ell}}+\delta .
\end{aligned}
$$

Thus

$S_{n}+I_{n}+P_{n} \leqslant \frac{\Lambda^{u}}{\mu^{\ell}}+\varepsilon+\frac{r^{u}+\gamma^{u} a^{u} f\left(\Lambda^{u} / \mu^{\ell}+\varepsilon, 0,0\right)+\theta^{u} \eta^{u} g_{0}\left(0, \Lambda^{u} / \mu^{\ell}+\varepsilon\right)\left(\Lambda^{u} / \mu^{\ell}+\varepsilon\right)}{b^{\ell}}+\delta$, and the result follows.

To formulate our next assumption we need to consider the system

$$
\left\{\begin{array}{l}
x_{n+1}-x_{n}=\Lambda_{n}-\mu_{n} x_{n+1}-a_{n} f\left(x_{n+1}, 0, z_{n}\right) z_{n} \\
z_{n+1}-z_{n}=\left(r_{n}-b_{n} z_{n+1}\right) z_{n}+\gamma_{n} a_{n} f\left(x_{n+1}, 0, z_{n}\right) z_{n}
\end{array} .\right.
$$

which corresponds to the dynamics of the susceptible preys and the predators in the absence of infected preys. We also need to consider the two families of auxiliary systems:

$$
\left\{\begin{array}{l}
x_{n+1}-x_{n}=\Lambda_{n}-\mu_{n} x_{n+1}-a_{n} f\left(x_{n+1}, 0, z_{n}\right) z_{n}-\varepsilon x_{n} \\
z_{n+1}-z_{n}=\left(r_{n}-b_{n} z_{n+1}\right) z_{n}+\gamma_{n} a_{n} f\left(x_{n+1}, \varepsilon, z_{n}\right) z_{n}
\end{array}\right.
$$

and

$$
\left\{\begin{array}{l}
x_{n+1}-x_{n}=\Lambda_{n}-\mu_{n} x_{n+1}-a_{n} f\left(x_{n+1}, \varepsilon, z_{n}\right) z_{n} \\
z_{n+1}-z_{n}=\left(r_{n}-b_{n} z_{n+1}\right) z_{n}+\gamma_{n} a_{n} f\left(x_{n+1}, 0, z_{n}\right) z_{n}+\theta_{n} \eta_{n} g\left(x_{n+1}, 0, z_{n}\right) \varepsilon
\end{array} .\right.
$$

We make the following assumptions concerning systems (13) and (14). 
H8) There is a family of nonnegative solutions $\left(x_{1, \varepsilon, n}^{*}, z_{1, \varepsilon, n}^{*}\right)$ of system (13), one for each $\varepsilon>0$ sufficiently small, such that each solution in the family is globally asymptotically stable in a set containing $\left\{(x, y) \in\left(R_{0}^{+}\right)^{2}: x, z>0\right\}$ and the function $\varepsilon \mapsto\left(x_{1, \varepsilon, n}^{*}, z_{1, \varepsilon, n}^{*}\right)$ is continuous.

H9) There is a family of nonegative solutions $\left(x_{2, \varepsilon, n}^{*}, z_{2, \varepsilon, n}^{*}\right)$ of system (14), one for each $\varepsilon>0$ sufficiently small, such that each solution in the family is globally asymptotically stable in a set containing $\left\{(x, y) \in\left(R_{0}^{+}\right)^{2}: x, z>0\right\}$ and the function $\varepsilon \mapsto\left(x_{2, \varepsilon, n}^{*}, z_{2, \varepsilon, n}^{*}\right)$ is continuous.

We denote the element of the family of solutions in H8) (or H9)) with $\varepsilon=0$, by $\left(x_{n}^{*}, z_{n}^{*}\right)$. For each solution $\left(x_{n}^{*}, z_{n}^{*}\right)$ of (13) with $\varepsilon=0$ and initial conditions $\left(x_{0}, y_{0}\right)$ with $x_{0}>0$ and $z_{0}>0$, and each $\lambda \in \mathbb{N}$, define the number

$$
\mathcal{R}^{\ell}(\lambda)=\liminf _{n \rightarrow+\infty} \prod_{k=n}^{n+\lambda} \frac{1+\beta_{k} x_{k+1}^{*}}{1+c_{k}+\eta_{k} g\left(x_{k}^{*}, 0, z_{k}^{*}\right)}
$$

and for each solution $\left(s_{n}^{*}\right)$ of (5) with $s_{0}>0$, each solution $\left(y_{n}^{*}\right)$ of (므) with $y_{0}>0$ and each $\lambda \in \mathbb{N}$, define the number

$$
\mathcal{R}^{u}(\lambda)=\limsup _{n \rightarrow+\infty} \prod_{k=n}^{n+\lambda} \frac{1+\beta_{k} s_{k+1}^{*}}{1+c_{k}+\eta_{k} g\left(s_{k}^{*}, 0, y_{k}^{*}\right)}
$$

These numbers will be useful in obtaining conditions for permanence and extinction and, in some sense, play the role of upper and lower bounds for the basic reproductive number in this general context. In the following lemma we prove that the numbers above are independent of the particular positive solutions of (5), (8) and (13) considered.

Lemma 4. The numbers $\mathcal{R}^{\ell}(\lambda)$ and $\mathcal{R}^{u}(\lambda)$ are independent of the particular solutions $\left(s_{n}^{*}\right)$ of (5) with $s_{0}>0,\left(y_{n}^{*}\right)$ of (8) with $y_{0}>0$ and $\left(x_{n}^{*}, z_{n}^{*}\right)$ of (12) with $x_{0}>0$ and $z_{0}>0$.

Proof. Write $\mathcal{R}^{\ell}(\lambda, x, z)$ for the number in (15) corresponding to the solution $(x, z)=$ $\left(x_{n}^{*}, z_{n}^{*}\right)_{n \in \mathbb{N}}$ of (12) with $x_{0}>0$ and $z_{0}>0$.

Let $\left(x_{1}^{*}, z_{1}^{*}\right)=\left(x_{1, n}^{*}, z_{1, n}^{*}\right)_{n \in \mathbb{N}}$ and $\left(x_{2}^{*}, z_{2}^{*}\right)=\left(x_{2, n}^{*}, z_{2, n}^{*}\right)_{n \in \mathbb{N}}$ be distinct solutions of (12) with $x_{1,0}>0, z_{1,0}>0, x_{2,0}>0$ and $z_{2,0}>0$.

Let $\delta>0$ be sufficiently small. By assumptions H8) (or H9)), for $k \geqslant N$ (where $N \in \mathbb{N}$ ) sufficiently large, we have

$$
x_{1, k}^{*}-\delta \leqslant x_{2, k}^{*} \leqslant x_{1, k}^{*}+\delta \quad \text { and } \quad z_{1, k}^{*}-\delta \leqslant z_{2, k}^{*} \leqslant z_{1, k}^{*}+\delta .
$$

Additionally, by $\mathrm{H}(3)$, there is $c>0$ such that, for sufficiently large $k$,

$$
\left|g\left(x_{1, k}^{*}, 0, z_{1, k}^{*}\right)-g\left(x_{1, k}^{*}, 0, z_{2, k}^{*}-\delta\right)\right| \leqslant c\left|z_{1, k}^{*}-z_{2, k}^{*}+\delta\right| \leqslant 2 c \delta
$$

and

$$
\left|g\left(x_{2, k}^{*}, 0, z_{1, k}^{*}\right)-g\left(x_{1, k}^{*}, 0, z_{1, k}^{*}\right)\right| \leqslant c\left|z_{1, k}^{*}-z_{2, k}^{*}\right| \leqslant c \delta .
$$


Thus, for $n \geqslant N$

$$
\begin{aligned}
& \prod_{k=n}^{n+\lambda} \frac{1+\beta_{k} x_{2, k+1}^{*}}{1+c_{k}+\eta_{k} g\left(x_{2, k}^{*}, 0, z_{2, k}^{*}\right)} \\
& \leqslant \prod_{k=n}^{n+\lambda} \frac{1+\beta_{k} x_{1, k+1}^{*}+\delta \beta_{k}}{1+c_{k}+\eta_{k} g\left(x_{2, k}^{*}, 0, z_{1, k}^{*}-\delta\right)} \\
& \leqslant \prod_{k=n}^{n+\lambda} \frac{1+\beta_{k} x_{1, k+1}^{*}+\delta \beta_{k}}{1+c_{k}+\eta_{k} g\left(x_{1, k}^{*}, 0, z_{1, k}^{*}\right)} \frac{1+c_{k}+\eta_{k} g\left(x_{1, k}^{*}, 0, z_{1, k}^{*}\right)}{1+c_{k}+\eta_{k} g\left(x_{2, k}^{*}, 0, z_{1, k}^{*}-\delta\right)} \\
& =\prod_{k=n}^{n+\lambda}\left(\frac{1+\beta_{k} x_{1, k+1}^{*}}{1+c_{k}+\eta_{k} g\left(x_{1, k}^{*}, 0, z_{1, k}^{*}\right)}+\frac{\delta \beta_{k}}{1+c_{k}+\eta_{k} g\left(x_{1, k}^{*}, 0, z_{1, k}^{*}\right)}\right) \times \\
& \times\left(1+\frac{3 c \delta \eta_{k}}{1+c_{k}+\eta_{k} g\left(x_{2, k}^{*}, 0, z_{2, k}^{*}-\delta\right)}\right) \\
& \leqslant(1+\delta B)^{\lambda} \prod_{k=n}^{n+\lambda}\left(\frac{1+\beta_{k} x_{1, k+1}^{*}}{1+c_{k}+\eta_{k} g\left(x_{1, k}^{*}, 0, z_{1, k}^{*}\right)}+\delta A\right) \\
& \leqslant(1+\delta B)^{\lambda}\left(\prod_{k=n}^{n+\lambda} \frac{1+\beta_{k} x_{1, k+1}^{*}}{1+c_{k}+\eta_{k} g\left(x_{1, k}^{*}, 0, z_{1, k}^{*}\right)}+\sum_{j=1}^{\lambda+1}\left(\begin{array}{c}
\lambda+1 \\
j
\end{array}\right) \delta^{j} C^{\lambda+1-j} A^{j}\right) \text {, }
\end{aligned}
$$

where

$$
A=\frac{\beta^{u}}{1+c^{\ell}+\eta^{\ell}\left(g\left(x_{1, k}^{*}, 0, z_{1, k}^{*}\right)\right)^{\ell}}, \quad B=\frac{2 c \eta^{u}}{1+c^{\ell}+\eta^{\ell}\left(g\left(x_{1, k}^{*}, 0, z_{2, k}^{*}-\delta\right)\right)^{\ell}}
$$

and

$$
C=\frac{1+\beta^{u}\left(x_{1}^{*}\right)^{u}}{1+c^{\ell}+\eta^{\ell}\left(g\left(x_{1, k}^{*}, 0, z_{1, k}^{*}\right)\right)^{\ell}} .
$$

By (17), we conclude that

$$
\mathcal{R}^{\ell}\left(\lambda, x_{2}^{*}, z_{2}^{*}\right) \leqslant(1+\delta B)^{\lambda}\left(\mathcal{R}^{\ell}\left(\lambda, x_{1}^{*}, z_{1}^{*}\right)+\sum_{j=1}^{\lambda+1}\left(\begin{array}{c}
\lambda+1 \\
j
\end{array}\right) \delta^{j} C^{\lambda+1-j} A^{j}\right) .
$$

By the arbitrariness of $\delta>0$, we conclude that $\mathcal{R}^{\ell}\left(\lambda, x_{2}^{*}, z_{2}^{*}\right) \leqslant \mathcal{R}^{\ell}\left(\lambda, x_{1}^{*}, z_{1}^{*}\right)$ and, interchanging the roles of $\left(x_{1}^{*}, z_{1}^{*}\right)$ and $\left(x_{2}^{*}, z_{2}^{*}\right)$ it is immediate that $\mathcal{R}^{\ell}\left(\lambda, x_{2}^{*}, z_{2}^{*}\right) \geqslant$ $\mathcal{R}^{\ell}\left(\lambda, x_{1}^{*}, z_{1}^{*}\right)$. Thus $\mathcal{R}^{\ell}\left(\lambda, x_{2}^{*}, z_{2}^{*}\right)=\mathcal{R}^{\ell}\left(\lambda, x_{1}^{*}, z_{1}^{*}\right)$.

Now write $\mathcal{R}^{u}(\lambda, s, y)$ for the number in (16) corresponding to the solutions $s=\left(s_{n}^{*}\right)$ of (5) with $s_{0}>0$ and $y=\left(y_{n}^{*}\right)$ of (8) with $y_{0}>0$.

Let again $\delta>0$ be sufficiently small. Additionally, let $s_{1}^{*}=\left(s_{1, n}^{*}\right)$ and $s_{2}^{*}=\left(s_{2, n}^{*}\right)$ be distinct solutions of (5) and $y_{1}^{*}=\left(y_{1, n}^{*}\right)$ and $y_{2}^{*}=\left(y_{2, n}^{*}\right)$ be distinct solutions of (8). By iv) in Lemma 1 and iv) in Lemma 2, we have

$$
s_{1, k}^{*}-\delta \leqslant s_{2, k}^{*} \leqslant s_{2, k}^{*}+\delta \quad \text { and } \quad y_{1, k}^{*}-\delta \leqslant y_{2, k}^{*} \leqslant y_{1, k}^{*}+\delta
$$

for $k \geqslant N$ sufficiently large. There is $c>0$ such that

$$
\left|g\left(s_{1, k}^{*}, 0, y_{1, k}^{*}\right)-g\left(s_{1, k}^{*}, 0, y_{2, k}^{*}-\delta\right)\right| \leqslant c\left|y_{1, k}^{*}-y_{2, k}^{*}+\delta\right| \leqslant 2 c \delta
$$

and

$$
\left|g\left(s_{2, k}^{*}, 0, y_{1, k}^{*}\right)-g\left(s_{1, k}^{*}, 0, y_{1, k}^{*}\right)\right| \leqslant c\left|s_{2, k}^{*}-s_{1, k}^{*}\right| \leqslant c \delta .
$$


Therefore

$$
\begin{aligned}
& \prod_{k=n}^{n+\lambda} \frac{1+\beta_{k} s_{2, k+1}^{*}}{1+c_{k}+\eta_{k} g\left(s_{2, k}^{*}, 0, y_{2, k}^{*}\right)} \\
\leqslant & \prod_{k=n}^{n+\lambda} \frac{1+\beta_{k} s_{1, k+1}^{*}+\delta \beta_{k}}{1+c_{k}+\eta_{k} g\left(s_{2, k}^{*}, 0, y_{1, k}^{*}-\delta\right)} \\
\leqslant & \prod_{k=n}^{n+\lambda} \frac{1+\beta_{k} s_{1, k+1}^{*}+\delta \beta_{k}}{1+c_{k}+\eta_{k} g\left(s_{1, k}^{*}, 0, y_{1, k}^{*}\right)} \frac{1+c_{k}+\eta_{k} g\left(s_{1, k}^{*}, 0, y_{1, k}^{*}\right)}{1+c_{k}+\eta_{k} g\left(s_{2, k}^{*}, 0, y_{1, k}^{*}-\delta\right)} \\
\leqslant & \prod_{k=n}^{n+\lambda}\left(\frac{1+\beta_{k} s_{1, k+1}^{*}}{1+c_{k}+\eta_{k} g\left(s_{1, k}^{*}, 0, y_{1, k}^{*}\right)}+\frac{3 \beta_{k}}{1+c_{k}+\eta_{k} g\left(s_{1, k}^{*}, 0, y_{1, k}^{*}\right)}\right) \times \\
& \times\left(1+\frac{3 c \delta \eta_{k}}{1+c_{k}+\eta_{k} g\left(s_{2, k}^{*}, 0, y_{1, k}^{*}-\delta\right)}\right) \\
\leqslant & (1+\delta B)^{\lambda} \prod_{k=n}^{n+\lambda}\left(\frac{1+\beta_{k} s_{1, k+1}^{*}}{1+c_{k}+\eta_{k} g\left(s_{1, k}^{*}, 0, y_{1, k}^{*}\right)}+\delta A\right) \\
\leqslant & (1+\delta B)^{\lambda}\left(\prod_{k=n}^{n+\lambda} \frac{1+\beta_{k} s_{1, k+1}^{*}}{1+c_{k}+\eta_{k} g\left(s_{1, k}^{*}, 0, y_{1, k}^{*}\right)}+\sum_{j=1}^{\lambda+1}\left(\begin{array}{c}
\lambda+1 \\
j
\end{array}\right) \delta^{j} C^{\lambda+1-j} A^{j}\right),
\end{aligned}
$$

for $n \geqslant N$, where

$$
A=\frac{\beta^{u}}{1+c^{\ell}+\eta^{\ell}\left(g\left(s_{1, k}^{*}, 0, y_{1, k}^{*}\right)\right)^{\ell}}, \quad B=\frac{2 c \eta^{u}}{1+c^{\ell}+\eta^{\ell}\left(g\left(s_{2, k}^{*}, 0, y_{1, k}^{*}-\delta\right)\right)^{\ell}}
$$

and

$$
C=\frac{1+\beta^{u}\left(s_{1}^{*}\right)^{u}}{1+c^{\ell}+\eta^{\ell}\left(g\left(s_{1, k}^{*}, 0, y_{1, k}^{*}\right)\right)^{\ell}}
$$

By (18), we conclude that

$$
\mathcal{R}^{\ell}\left(\lambda, s_{2}^{*}, y_{2}^{*}\right) \leqslant(1+\delta B)^{\lambda}\left(\mathcal{R}^{\ell}\left(\lambda, s_{1}^{*}, y_{1}^{*}\right)+\sum_{j=1}^{\lambda+1}\left(\begin{array}{c}
\lambda+1 \\
j
\end{array}\right) \delta^{j} C^{\lambda+1-j} A^{j},\right)
$$

By the arbitrariness of $\varepsilon>0$, we conclude that $\mathcal{R}^{\ell}\left(\lambda, s_{2}^{*}, y_{2}^{*}\right) \leqslant \mathcal{R}^{\ell}\left(\lambda, s_{1}^{*}, y_{1}^{*}\right)$ and, interchanging the roles of $\left(s_{1}^{*}, y_{1}^{*}\right)$ and $\left(s_{2}^{*}, y_{2}^{*}\right)$ it is immediate that $\mathcal{R}^{\ell}\left(\lambda, s_{2}^{*}, y_{2}^{*}\right) \geqslant$ $\mathcal{R}^{\ell}\left(\lambda, s_{1}^{*}, y_{1}^{*}\right)$. Thus $\mathcal{R}^{\ell}\left(\lambda, s_{2}^{*}, y_{2}^{*}\right)=\mathcal{R}^{\ell}\left(\lambda, s_{1}^{*}, y_{1}^{*}\right)$.

The result is proved.

\section{EXTinCtion AND STRONG PERSistence}

In this section we establish our main results on extinction and persistence. To obtain our result on extinction we must make some additional assumptions on the function $g$. In spite of this, it is easy to see that the usual growth rates still fulfill these assumptions.

Theorem 1. Assume that $g(S+I, 0, P) \leqslant g(S, I, P)$. If there is $\lambda \in \mathbb{N}$ such that $\mathcal{R}^{u}(\lambda)<1$ then the infectives $\left(I_{n}\right)$ go to extinction in system (2). Furthermore, if $a \equiv 0$ and $g(S, I, P)=g_{0}(S, I) P$, any disease-free solution $\left(s_{n}^{*}, 0, y_{n}^{*}\right)$ of (2), where $\left(s_{n}^{*}\right)$ is a solution of (5) and $\left(y_{n}^{*}\right)$ is a solution of (8), is globally asymptotically attractive. 
Proof. Since $\mathcal{R}^{u}(\lambda)<1$, given $\delta_{1}>0$ sufficiently small, there are $\delta_{0}>0$ and $N \in \mathbb{N}$ such that

$$
\prod_{k=n}^{n+\lambda} \frac{1+\beta_{k}\left(s_{k+1}^{*}+\delta\right)}{\left.1+c_{k}+\eta_{k} g\left(s_{k}^{*}+\delta, 0, y_{k}^{*}-\delta\right)\right)}<1-\delta_{1},
$$

for $n \geqslant N$ and all positive $\delta \leqslant \delta_{0}$. Let $N_{n}=S_{n}+I_{n}$. Since $\mu_{n} \leqslant c_{n}$, by the first two equations in (2), we conclude that

$$
N_{n+1}-N_{n} \leqslant \Lambda_{n}-\mu_{n} N_{n+1} \quad \Leftrightarrow \quad N_{n+1} \leqslant \frac{\Lambda_{n}}{1+\mu_{n}}+\frac{N_{n}}{1+\mu_{n}}
$$

and thus $S_{n}, I_{n} \leqslant N_{n} \leqslant s_{n}$, where $\left(s_{n}\right)$ is any solution of (5) with $s_{0}=S_{0}$. By (iv) in Lemma 1 we have $\left|s_{n}-s_{n}^{*}\right| \leqslant \delta_{0}$ for sufficiently large $n$, say $n \geqslant N_{1} \geqslant N$. Thus

$$
S_{n}, I_{n} \leqslant S_{n}+I_{n}=N_{n} \leqslant s_{n} \leqslant s_{n}^{*}+\delta_{0},
$$

for $n \geqslant N_{1}$.

By the third equation in (2), we conclude that

$$
P_{n+1}-P_{n} \geqslant\left(r_{n}-b_{n} P_{n+1}\right) P_{n} \quad \Leftrightarrow \quad P_{n+1} \geqslant \frac{r_{n} P_{n}+P_{n}}{1+b_{n} P_{n}}
$$

and thus $P_{n} \geqslant y_{n}$, where $\left(y_{n}\right)$ is any solution of (5) with $y_{0}=P_{0}$. By iv in Lemma 2 we have $\left|y_{n}-y_{n}^{*}\right| \leqslant \delta_{0}$ for sufficiently large $n$, say $n \geqslant N_{2} \geqslant N_{1}$. Thus

$$
P_{n} \geqslant y_{n} \geqslant y_{n}^{*}-\delta_{0}
$$

for $n \geqslant N_{2}$. Using our hypothesis, by the second equation in (2) and (19),

$$
\begin{aligned}
I_{n+1} & =\frac{\beta_{n} S_{n+1} I_{n}+I_{n}}{1+\eta_{n} g\left(S_{n}, I_{n}, P_{n}\right)+c_{n}} \\
& \leqslant \frac{\beta_{n} S_{n+1} I_{n}+I_{n}}{1+\eta_{n} g\left(S_{n}+I_{n}, 0, P_{n}\right)+c_{n}} \\
& \leqslant \frac{\beta_{n}\left(s_{n+1}^{*}+\delta_{0}\right)+1}{1+c_{n}+\eta_{n} g\left(s_{n}^{*}+\delta_{0}, 0, y_{n}^{*}-\delta_{0}\right)} I_{n} \\
& <\left(1-\delta_{1}\right) I_{n-\lambda-1} \\
& <\cdots<\left(1-\delta_{1}\right)^{\lfloor n /(\lambda+1)\rfloor} I_{n-\lfloor n /(\lambda+1)\rfloor(\lambda+1)} \\
& \leqslant d\left(\left(1-\delta_{1}\right)^{1 /(\lambda+1)}\right)^{n},
\end{aligned}
$$

for $n \geqslant N_{2}$, where $d=\max _{j=0, \ldots, \lambda} I_{j}$. We conclude that $I_{n} \rightarrow 0$ as $n \rightarrow+\infty$ and we have extinction of the infectives.

Assume now that $a \equiv 0$ and $g(S, I, P)=g_{0}(S, I) P$, let $\left(\left(S_{n}, I_{n}, P_{n}\right)\right)$ be any solution of (2) and consider the sequence $\left(\left(s_{n}^{*}, 0, y_{n}^{*}\right)\right)$, where $\left(s_{n}^{*}\right)$ is a solution of (5) and $\left(y_{n}^{*}\right)$ is a solution of (8).

Since $I_{n} \rightarrow 0$ as $n \rightarrow+\infty$, given $\delta>0$ there is $T \in \mathbb{N}$ such that $I_{n}<\delta$ for $n \geqslant T$. Letting $U_{n}=S_{n}-s_{n}^{*}$, we have, by the first equation in (2),

$$
U_{n+1}-U_{n}=-\mu_{n} U_{n+1}-\beta_{n} S_{n+1} I_{n},
$$

for $n \geqslant T$. Thus, by iv) in Lemma 1 and by Lemma 3, we have

$$
-\beta^{u} L \delta<\left(1+\mu_{n}\right) U_{n+1}-U_{n}<0
$$

for $n$ sufficiently large. 
We get, for $\delta>0$ sufficiently small

$$
\begin{aligned}
U_{n+1} & >-\frac{\beta^{u} L \delta}{1+\mu_{n}}+\frac{1}{1+\mu_{n}} U_{n} \\
& >-\frac{\beta^{u} L \delta}{1+\mu_{n}}+\frac{1}{1+\mu_{n}}\left(-\frac{\beta^{u} K \delta}{1+\mu_{n-1}}+\frac{1}{1+\mu_{n-1}} U_{n-1}\right) \\
& >\cdots \\
& >\left(\prod_{m=0}^{n-1} \frac{1}{1+\mu_{m}}\right) U_{0}-\sum_{m=0}^{n-1}\left(\beta^{u} L \delta\right)^{m+1}\left(\prod_{k=m}^{n-1} \frac{1}{1+\mu_{k}}\right) \\
& >\left(\prod_{m=0}^{n-1} \frac{1}{1+\mu_{m}}\right) U_{0}-\delta \beta^{u} L \sum_{m=0}^{n-1} K \theta^{n-m} \\
& >\left(\prod_{m=0}^{n-1} \frac{1}{1+\mu_{m}}\right) U_{0}-\frac{\beta^{u} L K \theta}{1-\theta} \delta \\
& >-\frac{\beta^{u} L K \theta}{1-\theta} \delta .
\end{aligned}
$$

Similarly,

$$
U_{n+1}<\frac{1}{1+\mu_{n}} U_{n}<\left(\prod_{m=0}^{n-1} \frac{1}{1+\mu_{m}}\right) U_{0} .
$$

Since

$$
\prod_{m=0}^{n-1} \frac{1}{1+\mu_{m}} \rightarrow 0 \quad \text { as } \quad n \rightarrow+\infty
$$

given $\delta>0$, we have $\left|U_{n+1}\right|<M \delta$, where $M=\beta^{u} L K \theta /(1-\theta)$, for sufficiently large $n$. We conclude that $\left|U_{n}\right| \rightarrow 0$ as $n \rightarrow+\infty$ and thus

$$
S_{n} \rightarrow s_{n}^{*} \quad \text { as } \quad n \rightarrow+\infty \text {. }
$$

By the third equation in (2), we have, for sufficiently large $n$,

$$
\begin{aligned}
P_{n+1}-P_{n} & =\left(r_{n}-b_{n} P_{n+1}\right) P_{n}+\theta_{n} \eta_{n} g_{0}\left(S_{n}, I_{n}\right) P_{n} I_{n+1} \\
& \leqslant\left(r_{n}-b_{n} P_{n+1}\right) P_{n}+\theta_{n} \eta_{n} g_{0}\left(S_{n}+I_{n}, 0\right) P_{n} I_{n+1} \\
& \leqslant\left(r_{n}-b_{n} P_{n+1}\right) P_{n}+\theta_{n} \eta_{n} g_{0}\left(s_{n}^{*}+2 \delta, 0\right) P_{n} \delta
\end{aligned}
$$

and thus

$$
\left(r_{n}-b_{n} P_{n+1}\right) P_{n} \leqslant P_{n+1}-P_{n} \leqslant\left(r_{n}+\theta^{u} \eta^{u} g_{0}\left(s_{n}^{*}+2 \delta, 0\right) \delta-b_{n} P_{n+1}\right) P_{n} .
$$

We conclude that

$$
\frac{r_{n} P_{n}+P_{n}}{1+b_{n} P_{n}} \leqslant P_{n+1} \leqslant \frac{\left(r_{n}+\theta^{u} \eta^{u} g_{0}\left(s_{n}^{*}+2 \delta, 0\right) \delta\right) P_{n}+P_{n}}{1+b_{n} P_{n}} .
$$

By ( $)$ in Lemma 2, we have $\left|P_{n}-y_{n}^{*}\right| \rightarrow 0$ as $n \rightarrow+\infty$. The result follows since $\left(S_{n}, I_{n}, P_{n}\right) \rightarrow\left(s_{n}^{*}, 0, y_{n}^{*}\right)$ as $n \rightarrow+\infty$.

Theorem 2. If there is a constant $\lambda \in \mathbb{N}$ such that $\mathcal{R}^{\ell}(\lambda)>1$ then the infectives $\left(I_{n}\right)$ are strong persistent in system (2). 
Proof. Assume that there is a constant $\lambda>0$ such that $\mathcal{R}^{\ell}(\lambda)>1$. Then, there is a function $\psi$ such that, for all $\delta>0$ sufficiently small we have

$$
\prod_{k=n}^{n+\lambda} \frac{1+\beta_{k}\left(x_{k+1}^{*}-\delta_{0}\right)}{1+c_{k}+\eta_{k} g\left(s_{k}^{*}-\delta_{0}, 0, z_{k}^{*}+\delta_{0}\right)}>1+\psi(\delta),
$$

with $\psi(\delta)>0$ for all $\delta>0$ and $\psi(\delta) \rightarrow 0$ as $\delta \rightarrow 0$. Let $N_{1} \in \mathbb{N}$ and $\left(S_{n}, I_{n}, P_{n}\right)$ be a solution of (2) with $I_{n}>0$ for all $n \geqslant N_{1}$. We will use a contradiction argument to prove that there is $\varepsilon_{1}>0$ such that

$$
\limsup _{n \rightarrow+\infty} I_{n}>\varepsilon_{1}
$$

We may assume that $\varepsilon_{1}>0$ is sufficiently small so that H8) and H9) hold for $\varepsilon_{1}$. Assuming that (22) does not hold, there is $N_{2} \geqslant N_{1}$ such that $I_{n}<\varepsilon_{1}$ for all $n \geqslant N_{2}$. By the first and third equation in (21), we conclude that

$$
\left\{\begin{array}{l}
S_{n+1}-S_{n} \leqslant \Lambda_{n}-\mu_{n} S_{n+1}-a_{n} f\left(S_{n+1}, \varepsilon_{1}, P_{n}\right) P_{n} \\
P_{n+1}-P_{n} \leqslant\left(r_{n}-b_{n} P_{n+1}\right) P_{n}+\gamma_{n} a_{n} f\left(S_{n+1}, 0, P_{n}\right) P_{n}+\theta_{n} \eta_{n} g\left(S_{n}, 0, P_{n}\right) P_{n} \varepsilon_{1}
\end{array},\right.
$$

for all $n \geqslant N_{2}$. Considering system (14) with $\varepsilon=\varepsilon_{1}$, we have $S_{n} \leqslant x_{2, \varepsilon_{1}, n}$ and $P_{n} \leqslant z_{2, \varepsilon_{1}, n}$ for sufficiently large $n$. By H9) we also have, for sufficiently large $n$,

$$
x_{2, \varepsilon_{1}, n} \leqslant x_{2, \varepsilon_{1}, n}^{*}+\varepsilon_{1} \quad \text { and } \quad z_{2, \varepsilon_{1}, n} \leqslant z_{2, \varepsilon_{1}, n}^{*}+\varepsilon_{1}
$$

and by the continuity properties in $\mathrm{H}(8)$ and $\mathrm{H}(9)$, we have

$x_{2, \varepsilon_{1}, n} \leqslant x_{2, \varepsilon_{1}, n}^{*}+\varepsilon_{1} \leqslant x_{2, n}^{*}+\chi_{1}\left(\varepsilon_{1}\right) \quad$ and $\quad z_{2, \varepsilon_{1}, n} \leqslant z_{2, \varepsilon_{1}, n}^{*}+\varepsilon_{1} \leqslant z_{2, \varepsilon_{1}, n}^{*}+\chi_{2}\left(\varepsilon_{1}\right)$, with $\chi_{1}\left(\varepsilon_{1}\right), \chi_{2}\left(\varepsilon_{1}\right) \rightarrow 0$ as $\varepsilon_{1} \rightarrow 0$. Thus, in particular, for sufficiently large $n$,

$$
S_{n} \leqslant x_{2, \varepsilon, n}^{*} \leqslant x_{2, n}^{*}+\chi_{1}\left(\varepsilon_{1}\right) \quad \text { and } \quad P_{n} \leqslant z_{2, \varepsilon, n}^{*} \leqslant z_{2, \varepsilon_{1}, n}^{*}+\chi_{2}\left(\varepsilon_{1}\right),
$$

Again by the first and third equation in (2), we conclude that

$$
\left\{\begin{array}{l}
S_{n+1}-S_{n} \geqslant \Lambda_{n}-\mu_{n} S_{n+1}-a_{n} f\left(S_{n+1}, 0, P_{n}\right) P_{n}-\beta_{n} S_{n+1} \varepsilon_{1} \\
P_{n+1}-P_{n} \geqslant\left(r_{n}-b_{n} P_{n+1}\right) P_{n}+\gamma_{n} a_{n} f\left(S_{n+1}, \varepsilon_{1}, P_{n}\right) P_{n}
\end{array},\right.
$$

for all $n \geqslant N_{2}$.

Consider system (13) with $\varepsilon=\varepsilon_{1}$. We have $S_{n} \geqslant x_{1, \varepsilon_{1}, n}$ and $P_{n} \geqslant z_{1, \varepsilon_{1}, n}$ for sufficiently large $n$. By $\mathrm{H} 8$ ) we also have, for sufficiently large $n$,

$$
x_{1, \varepsilon_{1}, n} \geqslant x_{1, \varepsilon_{1}, n}^{*}-\varepsilon_{1} \quad \text { and } \quad z_{1, \varepsilon_{1}, n} \geqslant z_{1, \varepsilon_{1}, n}^{*}-\varepsilon_{1} .
$$

and by the continuity properties in $\mathrm{H}(8)$ and $\mathrm{H}(9)$, we have

$x_{1, \varepsilon_{1}, n} \geqslant x_{1, \varepsilon_{1}, n}^{*}-\varepsilon_{1} \geqslant x_{1, n}^{*}-\varphi_{1}\left(\varepsilon_{1}\right) \quad$ and $\quad z_{1, \varepsilon_{1}, n} \geqslant z_{1, \varepsilon_{1}, n}^{*}-\varepsilon_{1} \geqslant z_{1, \varepsilon_{1}, n}^{*}-\varphi_{2}\left(\varepsilon_{1}\right)$, with $\varphi_{1}\left(\varepsilon_{1}\right), \varphi_{2}\left(\varepsilon_{1}\right) \rightarrow 0$ as $\varepsilon_{1} \rightarrow 0$. Thus, in particular, for sufficiently large $n$,

$$
S_{n} \geqslant x_{1, \varepsilon, n} \geqslant x_{1, n}^{*}-\varphi_{1}\left(\varepsilon_{1}\right) \quad \text { and } \quad P_{n} \geqslant z_{1, \varepsilon, n} \geqslant z_{1, n}^{*}-\varphi_{2}\left(\varepsilon_{1}\right) .
$$

From the second equation in (2), (24), (23) and (21), we conclude that

$$
\begin{aligned}
I_{n+1} & =\frac{\beta_{n} S_{n+1} I_{n}+I_{n}}{1+\eta_{n} g\left(S_{n}, I_{n}, P_{n}\right)+c_{n}} \\
& \geqslant \frac{\beta_{n}\left(x_{1, \varepsilon_{1}, n}^{*}-\varepsilon_{1}\right)+1}{1+\eta_{n} g\left(x_{1, \varepsilon_{1}, n}^{*}-\varepsilon_{1}, 0, z_{2, \varepsilon_{1}, n}^{*}+\varepsilon_{1}\right)+c_{n}} I_{n} \\
& >\left(1+\psi\left(\varepsilon_{1}\right)\right) I_{n-\lambda-1} \\
& >\cdots>\left(1+\psi\left(\varepsilon_{1}\right)\right)^{\lfloor n /(\lambda+1)\rfloor} I_{n-\lfloor n /(\lambda+1)\rfloor(\lambda+1)},
\end{aligned}
$$


for all $n \geqslant N_{3}$ with $N_{3} \geqslant N_{2}$. Therefore, by (21) and (25), we conclude that $I_{n} \rightarrow+\infty$. A contradiction to Lemma 3. We have (22) and the infectives in system (2) are weak persistent.

Using again a contradiction argument, we will prove that we have strong persistence of the infectives. We may assume, with no loss of generality, that there are $\delta, \delta_{0}>0$ such that

$$
\prod_{k=n}^{n+\lambda} \frac{1+\beta_{k}\left(x_{k+1}^{*}-\delta_{0}\right)}{1+c_{k}+\eta_{k} g\left(x_{k}^{*}-\delta_{0}, 0, z_{k}^{*}+\delta_{0}\right)}>1+\delta,
$$

for all sufficiently large $n \in \mathbb{N}$. For each $z_{0}=\left(S_{0}, I_{0}, P_{0}\right)$, denote by $\left(\left(S_{n, z_{0}}, I_{n, z_{0}}, P_{n, z_{0}}\right)\right)$ the solution of (2) with $\left(S_{0, z_{0}}, I_{0, z_{0}}, P_{0, z_{0}}\right)=\left(S_{0}, I_{0}, P_{0}\right)$.

Proceeding by contradiction, if the system is not strong persistent, then there is a sequence of initial values $z_{0, k}=\left(S_{0, k}, I_{0, k}, P_{0, k}\right), k \in \mathbb{N}$, such that

$$
\liminf _{n \rightarrow+\infty} I_{n, z_{0, k}}<\frac{\varepsilon_{0}}{k^{2}} .
$$

From (22) and (26), for each $k \in \mathbb{N}$ there are sequences $\left(s_{m, k}\right)$ and $\left(t_{m, k}\right)$ such that

$$
\begin{gathered}
0<s_{1, k}<t_{1, k}<s_{2, k}<t_{2, k}<\cdots<s_{m, k}<t_{m, k}<\cdots, \\
s_{m, k} \rightarrow+\infty \text { as } m \rightarrow+\infty \\
I_{s_{m, k}, z_{0, k}}>\frac{\varepsilon_{0}}{k}, \quad I_{t_{m, k}, z_{0, k}}<\frac{\varepsilon_{0}}{k^{2}}
\end{gathered}
$$

and

$$
\frac{\varepsilon_{0}}{k^{2}} \leqslant I_{n, z_{0, k}} \leqslant \frac{\varepsilon_{0}}{k}, \text { for all } n \in\left[s_{m, k}, t_{m, k}-1\right] \cap \mathbb{N} .
$$

For any $n \in\left[s_{m, k}, t_{m, k}-1\right] \cap \mathbb{N}$ sufficiently large, we have, using (11),

$$
\begin{aligned}
I_{n+1, z_{0, k}} & =\frac{1+\beta_{n} S_{n+1, z_{0, k}}}{1+c_{n}+\eta_{n} g\left(S_{n, z_{0, k}}, I_{n, z_{0, k}}, P_{n, z_{0, k}}\right)} I_{n, z_{0, k}} \\
& >\frac{1}{1+c_{n}+\eta_{n} g\left(S_{n, z_{0, k}}, 0, P_{n, z_{0, k}}\right)} I_{n, z_{0, k}} \\
& \geqslant \frac{1}{1+a} I_{n, z_{0, k}},
\end{aligned}
$$

where $a=c^{u}+\eta^{u} g(0,0, L+\delta)>0$. Therefore, by (30), we obtain

$$
\frac{\varepsilon_{0}}{k^{2}}>I_{t_{m, k}, z_{0, k}} \geqslant\left(\frac{1}{1+a}\right)^{t_{m, k}-s_{m, k}} I_{s_{m, k}, z_{0, k}}>\left(\frac{1}{1+a}\right)^{t_{m, k}-s_{m, k}} \frac{\varepsilon_{0}}{k},
$$

and therefore we get

$$
t_{m, k}-s_{m, k}>\frac{\ln k}{\ln (1+a)} \rightarrow+\infty \quad \text { as } k \rightarrow+\infty .
$$

We conclude that we can choose $k_{1} \in \mathbb{N}$ such that

$$
t_{m, k}-s_{m, k}>n_{1}+\lambda+1,
$$

for all $k \geqslant k_{1}$.

Now, for all $k \geqslant k_{1}$ and $n \in\left[s_{m, k}+1, t_{m, k}\right] \cap \mathbb{N}$, we have

$$
\left\{\begin{aligned}
S_{n+1, z_{0, k}}-S_{n, z_{0, k}} \leqslant & \Lambda_{n}-\mu_{n} S_{n+1, z_{0, k}}-a_{n} f\left(S_{n+1, z_{0, k}}, P_{n, z_{0, k}}\right) P_{n, z_{0, k}} \\
P_{n+1, z_{0, k}}-P_{n, z_{0, k}} \leqslant & \left(r_{n}-b_{n} P_{n+1, z_{0, k}}\right) P_{n, z_{0, k}} \\
& +\gamma_{n} a_{n} f\left(S_{n+1, z_{0, k}}, P_{n, z_{0, k}}\right) P_{n, z_{0, k}}+\theta_{n} \eta_{n} g\left(S_{n, z_{0, k}}, I_{n, z_{0, k}}, P_{n, z_{0, k}}\right) \varepsilon_{1}
\end{aligned}\right.
$$


Let $\left(\bar{x}_{n}, \bar{z}_{n}\right)$ be a solution of (12) with initial condition $\bar{x}_{s_{m, k}+1}=S_{s_{m, k}+1}$ and $\bar{z}_{s_{m, k}+1}=P_{s_{m, k}+1}$. By H8), for sufficiently large $k \in \mathbb{N}$ we have

$$
\left|S_{n, z_{0}, k}-x_{n}^{*}\right| \leqslant\left|S_{n, z_{0}, k}-\bar{x}_{n}\right|+\left|\bar{x}_{n}-x_{n}^{*}\right|<\varepsilon_{0} / 2+\varepsilon_{0} / 2=\varepsilon_{0}
$$

for all $n \in\left[s_{m, k}+1, t_{m, k}\right] \cap \mathbb{N}$. In particular

$$
S_{n, z_{0}, k} \geqslant x_{n}^{*}-\varepsilon_{0},
$$

for all $n \in\left[s_{m, k}+1, t_{m, k}\right] \cap \mathbb{N}$. In a similar way, using H9), we conclude that, for sufficiently large $k \in \mathbb{N}$ we have

$$
P_{n, z_{0}, k} \leqslant y_{n}^{*}+\varepsilon_{0},
$$

for all $n \in\left[s_{m, k}+1, t_{m, k}\right] \cap \mathbb{N}$.

Finally, we have

$$
\begin{aligned}
I_{n+1, z_{0, k}} & =\frac{1+\beta_{n} S_{n+1, z_{0, k}}}{1+c_{n}+\eta_{n} g\left(S_{n, z_{0, k}}, I_{n, z_{0, k}}, P_{n, z_{0, k}}\right)} I_{n, z_{0, k}} \\
& \geqslant \frac{1+\beta_{n}\left(x_{n}^{*}-\varepsilon_{0}\right)}{1+c_{n}+\eta_{n} g\left(x_{n}^{*}-\varepsilon_{0}, 0, y_{n}^{*}+\varepsilon_{0}\right)} I_{n, z_{0, k}}
\end{aligned}
$$

for all $n \in\left[s_{m, k}+n_{1}+1, t_{m, k}\right] \cap \mathbb{N}$ and $k \geqslant n_{4}$. By (27) and (34) we get

$\frac{\varepsilon_{0}}{k^{2}}>I_{t_{m, k}, z_{0, k}} \geqslant I_{t_{m, k}-\lambda, z_{0, k}} \prod_{n=t_{m, k}-\lambda}^{t_{m, k}} \frac{1+\beta_{n}\left(x_{n}^{*}-\varepsilon_{0}\right)}{1+c_{n}+\eta_{n} g\left(x_{n}^{*}-\varepsilon_{0}, 0, y_{n}^{*}+\varepsilon_{0}\right)} I_{n, z_{0, k}}>\frac{\varepsilon_{0}}{k^{2}}$,

a contradiction. Thus we conclude that the infectives are strong persistent and the result follow.

\section{EXAMPLES}

4.1. A model with no predation of uninfected preys. Letting $a \equiv 0$ and $g(x, y, z)=z$ in (2), we obtain the model below that corresponds to the discrete counterpart of the model in 20].

$$
\left\{\begin{array}{l}
S_{n+1}-S_{n}=\Lambda_{n}-\mu_{n} S_{n+1}-\beta_{n} S_{n+1} I_{n} \\
I_{n+1}-I_{n}=\beta_{n} S_{n+1} I_{n}-\eta_{n} P_{n} I_{n+1}-c_{n} I_{n+1} \\
P_{n+1}-P_{n}=\left(r_{n}-b_{n} P_{n+1}\right) P_{n}+\theta_{n} \eta_{n} P_{n} I_{n+1}
\end{array} .\right.
$$

For model (35) we assume conditions H(1), H(2) and H(4). Notice that H(3) is trivial, H(5) and H(6) follow from the discussion on (4) with $a_{n}=0$, H(7) follows from Lemma 3 and $\mathrm{H} 8$ ) and $\mathrm{H}$ (9) follow from Lemma 1 and Lemma 2 respectively.

For each solution $\left(s_{n}^{*}\right)$ of (5) with $s_{0}>0$, each solution $\left(y_{n}^{*}\right)$ of (8) with $y_{0}>0$ and each $\lambda \in \mathbb{N}$, in this context of no predation (of uninfected preys) we set

$$
\mathcal{R}_{N P}^{\ell}(\lambda)=\liminf _{n \rightarrow+\infty} \prod_{k=n}^{n+\lambda} \frac{1+\beta_{k} s_{k+1}^{*}}{1+c_{k}+\eta_{k} y_{k}^{*}}
$$

and

$$
\mathcal{R}_{N P}^{u}(\lambda)=\limsup _{n \rightarrow+\infty} \prod_{k=n}^{n+\lambda} \frac{1+\beta_{k} s_{k+1}^{*}}{1+c_{k}+\eta_{k} y_{k}^{*}} .
$$

The next theorems correspond to discrete counterparts of the results in [20]. 

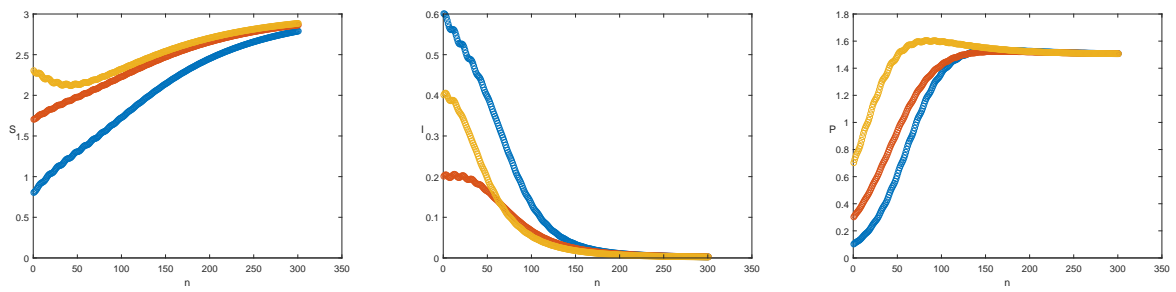

Figure 1. Extinction, when $\beta_{0}=0.17$.
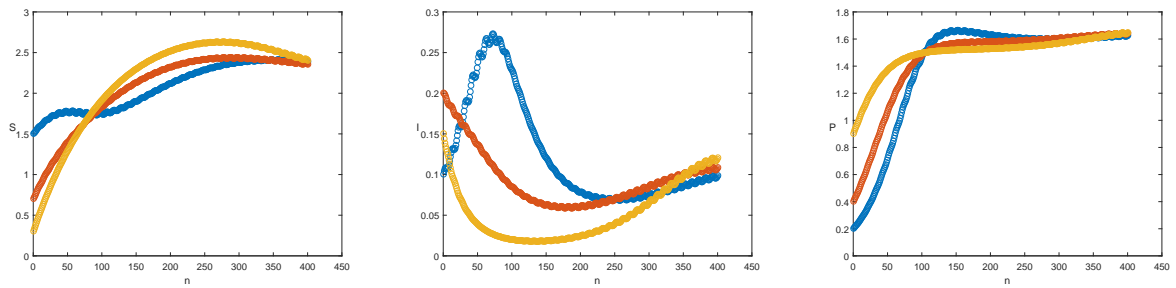

FiguRE 2. Strong persistence, when $\beta_{0}=0.29$.

Theorem 3. If there is $\lambda \in \mathbb{N}$ such that $\mathcal{R}_{N P}^{u}(\lambda)<1$ then the infectives $\left(I_{n}\right)$ go to extinction in system (35) and any disease-free solution $\left(\left(s_{n}^{*}, 0, y_{n}^{*}\right)\right)$ of (35), where $\left(s_{n}^{*}\right)$ is a solution of (5) and $\left(y_{n}^{*}\right)$ is a solution of (8), is globally asymptotically attractive.

Theorem 4. If there is $\lambda \in \mathbb{N}$ such that $\mathcal{R}_{N P}^{\ell}(\lambda)>1$ then the infectives $\left(I_{n}\right)$ are strongly persistent in system (35).

To do some simulation, we consider the particular solutions $s_{n}^{*}=\Lambda / \mu, y_{n}^{*}=r / b$ and the following particular set of parameters in system (35): $\Lambda_{n}=0.3, \mu_{n}=0.1$, $\beta_{n}=\beta_{0}(1+0.7 \cos (\pi n / 5)), \eta_{n}=0.3(1+0.7 \cos (\pi n / 5)), c_{n}=0.18, r_{n}=0.3$, $b_{n}=0.2, \theta_{n}=0.9$. This example is based on a continuous-time example in [10].

When $\beta_{0}=0.17$ we obtain $\mathcal{R}_{N P}^{u}(\lambda) \approx 0.89<1$ and we conclude that we have the extinction (figure 1). When $\beta_{0}=0.29$ we obtain $\mathcal{R}_{N P}^{\ell}(\lambda) \approx 1.24>1$ and we conclude that the infectives are strongly persistent (figure 2).

In extinction and uniform strong persistence scenario we considered, respectively, the following initial conditions: $\left(S_{0}, I_{0}, P_{0}\right)=(0.8,0.6,0.1),\left(S_{0}, I_{0}, P_{0}\right)=$ $(1.7,0.2,0.3)$ and $\left(S_{0}, I_{0}, P_{0}\right)=(2.3,0.4,0.7) ;\left(S_{0}, I_{0}, P_{0}\right)=(1.5,0.1,0.2),\left(S_{0}, I_{0}, P_{0}\right)=$ $(0.7,0.2,0.4)$ and $\left(S_{0}, I_{0}, P_{0}\right)=(0.3,0.15,0.9)$.

4.2. Periodic model. Consider the system (21) and assume that there is $\omega \in \mathbb{N}$ such that $\Lambda_{n+\omega}=\Lambda_{n}, \mu_{n+\omega}=\mu_{n}, a_{n+\omega}=a_{n}, \beta_{n+\omega}=\beta_{n}, \eta_{n+\omega}=\eta_{n}, c_{n+\omega}=c_{n}$, $r_{n+\omega}=r_{n}, b_{n+\omega}=b_{n}, \gamma_{n+\omega}=\gamma_{n}$ and $\theta_{n+\omega}=\theta_{n}$, for all $n \in \mathbb{N}$. Conditions H(1) to $\mathrm{H}(3)$ and $\mathrm{H}(5)$ to $\mathrm{H}(8)$ are assumed; condition $\mathrm{H}(4)$ is trivial.

For each solution $\left(s_{n}^{*}\right)$ of (5) with $s_{0}>0$, each solution $\left(y_{n}^{*}\right)$ of (8) with $y_{0}>0$ and for each solution $\left(\left(x_{n}^{*}, z_{n}^{*}\right)\right)$ of (13) with $\varepsilon=0$ and initial conditions $x_{0}>0$ and $z_{0}>0$, and each $\lambda \in \mathbb{N}$, we set 

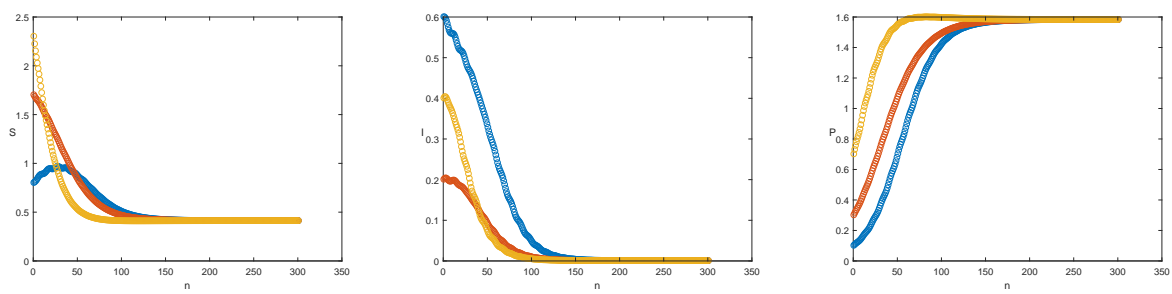

FiguRE 3. Extinction, when $\beta_{0}=0.17$.

$$
\mathcal{R}_{P E R}^{\ell}=\prod_{k=1}^{\omega} \frac{1+\beta_{k} x_{k+1}^{*}}{1+c_{k}+\eta_{k} g\left(x_{k}^{*}, 0, z_{k}^{*}\right)}
$$

and

$$
\mathcal{R}_{P E R}^{u}=\prod_{k=1}^{\omega} \frac{1+\beta_{k} s_{k+1}^{*}}{1+c_{k}+\eta_{k} g\left(s_{k}^{*}, 0, y_{k}^{*}\right)}
$$

Corollary 1. If $\mathcal{R}_{P E R}^{u}<1$ then the infective $\left(I_{n}\right)$ go to extinction in system (2) and any disease-free solution $\left(\left(s_{n}^{*}, 0, y_{n}^{*}\right)\right)$ of (2), where $\left(s_{n}^{*}\right)$ is a solution of the periodic version of (5) and $\left(y_{n}^{*}\right)$ is a solution of the periodic version of (8), is globally asymptotically attractive.

Corollary 2. If $\mathcal{R}_{P E R}^{\ell}>1$ then the infective $\left(I_{n}\right)$ is strongly persistent in system (2), where $\left(x_{n}^{*}\right)$ and $\left(z_{n}^{*}\right)$ are the components of the solution $\left(\left(x_{n}^{*}, z_{n}^{*}\right)\right)$ in the periodic version of (13). Moreover, there exist a periodic orbit of period $\omega$.

To do some simulation, we consider $f(x, y, z)=x, g(x, y, z)=z$, the particular solutions $s_{k}^{*}=\Lambda / \mu, y_{k}^{*}=r / b$ and

$$
\left(x_{n}^{*}, z_{n}^{*}\right)=\left(\frac{-K_{1}+\sqrt{K_{1}^{2}+2 \Lambda K_{2}}}{K_{2}},-\frac{K_{1}}{2 a}+\frac{1}{2 a} \sqrt{K_{1}^{2}+2 \Lambda K_{2}}+r / b\right),
$$

where $K_{1}=\mu+\frac{a r}{b}$ and $K_{2}=\frac{2 \gamma a^{2}}{b}$; we also considered the following particular set of parameters, and the exception of $\beta$ and $\eta$ we assume that they are all constants: $\Lambda_{n}=0.3, \mu_{n}=0.1, a_{n}=0.4, \beta_{n}=\beta_{0}(1+0.7 \cos (\pi n / 5)), \eta_{n}=$ $0.3(1+0.7 \cos (\pi n / 5)), c_{n}=0.18, r_{n}=0.3, b_{n}=0.2, \gamma_{n}=0.1$ and $\theta=0.9$.

When $\beta_{0}=0.17$, we obtain $\mathcal{R}_{P E R}^{u} \approx 0.44<1$ and we conclude that we have the extinction (figure 3). When $\beta_{0}=2.2$, we obtain $\mathcal{R}_{P E R}^{\ell}(\lambda) \approx 3.013>1$ and we conclude that the infectives are uniformly strong persistent (figure 4).

In extinction and uniform strong persistence scenario we considered, respectively, the following initial conditions: $\left(S_{0}, I_{0}, P_{0}\right)=(0.8,0.6,0.1),\left(S_{0}, I_{0}, P_{0}\right)=$ $(1.7,0.2,0.3)$ and $\left(S_{0}, I_{0}, P_{0}\right)=(2.3,0.4,0.7) ;\left(S_{0}, I_{0}, P_{0}\right)=(1.5,0.1,0.2),\left(S_{0}, I_{0}, P_{0}\right)=$ $(0.7,0.2,0.4)$ and $\left(S_{0}, I_{0}, P_{0}\right)=(0.3,0.15,0.9)$.

4.3. Autonomous model. Consider the system (2), and assume now that $f(x, y, z)=$ $x, g(x, y, z)=z, \Lambda_{n}=\Lambda, \mu_{n}=\mu, a_{n}=a, \beta_{n}=\beta, \eta_{n}=\eta, c_{n}=c, r_{n}=r, b_{n}=b$ and $\gamma_{n}=\gamma, \theta_{n}=\theta$. Then we obtain following the model: 

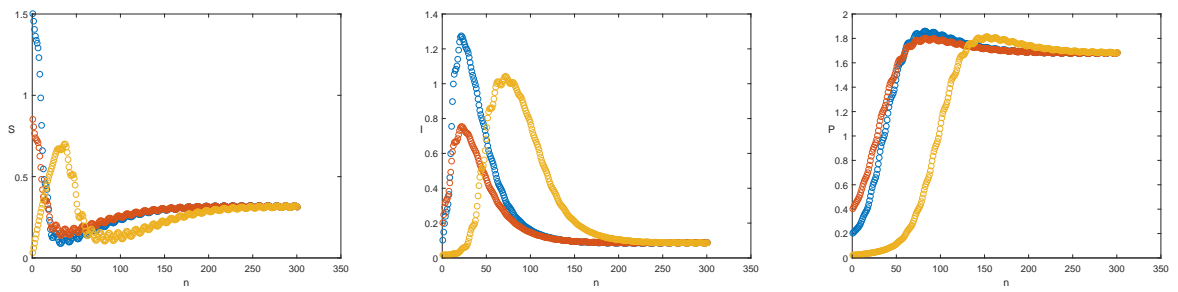

Figure 4. Strong persistence, when $\beta_{0}=2.2$.

$$
\left\{\begin{array}{l}
S_{n+1}-S_{n}=\Lambda-\mu S_{n+1}-a S_{n+1} P_{n}-\beta S_{n+1} I_{n} \\
I_{n+1}-I_{n}=\beta S_{n+1} I_{n}-\eta I_{n+1} P_{n}-c I_{n+1} \\
P_{n+1}-P_{n}=\left(r-b P_{n+1}\right) P_{n}+\gamma a S_{n+1} P_{n}+\theta \eta I_{n+1} P_{n}
\end{array} .\right.
$$

Conditions H(1) to H(4) are immediate. Conditions H(5) and H(6) follow from the discussion on (4). Condition H(7) follows from Lemma 3 and H(8) and H(9) follow from Lemma 1 and Lemma 2, respectively.

For each solution $\left(s_{n}^{*}\right)$ of (5) with $s_{0}>0$, each solution $\left(y_{0}^{*}\right)$ of (8) with $y_{0}>0$ and each solution $\left(\left(x_{n}^{*}, z_{n}^{*}\right)\right)$ of (13) with $\varepsilon=0$ and initial conditions $x_{0}>0$ and $z_{0}>0$, and each $\lambda \in \mathbb{N}$, we set

$$
\mathcal{R}_{A}^{\ell}=\frac{1+\beta\left(\frac{-K_{1}+\sqrt{K_{1}^{2}+2 \Lambda K_{2}}}{K_{2}}\right)}{1+c+\eta\left(-\frac{K_{1}}{2 a}+\frac{1}{2 a} \sqrt{K_{1}^{2}+2 \Lambda K_{2}}+r / b\right)}
$$

and

$$
\mathcal{R}_{A}^{u}=\frac{1+\beta(\Lambda / \mu)}{1+c+\eta(r / b)},
$$

where $K_{1}=\mu+\frac{a r}{b}$ and $K_{2}=\frac{2 \gamma a^{2}}{b}$.

Corollary 3. If $\mathcal{R}_{A}^{u}<1$ then the infective $\left(I_{n}\right)$ in system (36) go to extinction.

Corollary 4. If $\mathcal{R}_{A}^{\ell}>1$ then the infective $\left(I_{n}\right)$ in system (36) are strongly persistent.

To do some simulation, we consider the following particular set of parameters: $\Lambda=0.3, \mu=0.1, a=0.4, \eta=0.3, c=0.18, r=0.3, b=0.2, \gamma=0.1$ and $\theta=0.9$.

When $\beta=0.17$ we obtain $\mathcal{R}_{A}^{u} \approx 0.93<1$ and we conclude that we have the extinction. When $\beta=2.2$ we obtain $\mathcal{R}_{A}^{\ell}(\lambda) \approx 1.14>1$ and we conclude that the infectives are strongly persistent.

In uniform strong persistence and extinction scenario we considered, respectively, the following initial conditions: $\left(S_{0}, I_{0}, P_{0}\right)=(0.8,0.6,0.1),\left(S_{0}, I_{0}, P_{0}\right)=$ $(1.7,0.2,0.3)$ and $\left(S_{0}, I_{0}, P_{0}\right)=(2.3,0.4,0.7) ;\left(S_{0}, I_{0}, P_{0}\right)=(1.5,0.1,0.2),\left(S_{0}, I_{0}, P_{0}\right)=$ $(0.7,0.2,0.4)$ and $\left(S_{0}, I_{0}, P_{0}\right)=(0.3,0.15,0.9)$.

\section{REFERENCES}

[1] H. Bai and R. Xo, Global stability of a delayed eco-epidemiological model with holling type III functional response, Springer Proceedings in mathematics ans Statistics 225 (2018), 119-130. 

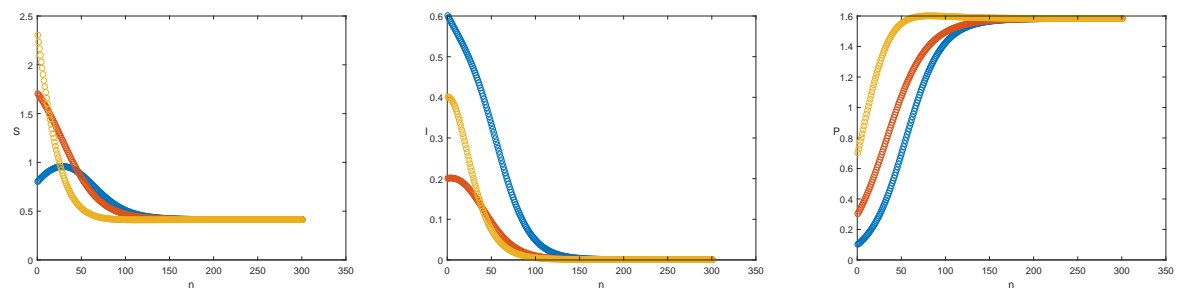

FiguRE 5. Extinction, when $\beta=0.17$.
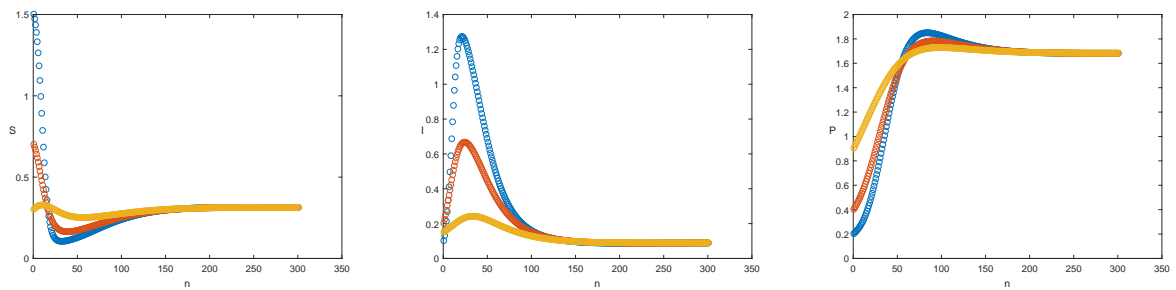

FIgURE 6. Strong persistence, when $\beta=2.2$.

[2] Chakraborty, K., Das K., Haldar, S., Kar,T.K, A mathematical study of an ecoepidemiological system on disease persistence and existinction perspective, Appl. Math. and Comput. 254 (2015), 99-112.

[3] A. P. Dobson, The population biology of parasite-induced changes in host behavior, Q. Rev. Biol. 30 (1988), 139-165.

[4] M. Friend, Avian disease at the Salton Sea, Hydrobiologia, 161 (2002), 293-306.

[5] M. Garrione and C. Rebelo, Persistence in seasonally varying predator-prey systems via the basic reproduction, Nonlinear Anal. Real World Appl. 30 (2016), 73-98.

[6] H. W. Hethcote, W. Wang, L. Han and Z. Ma, A predator-prey model with infected prey,Theor. Popul. Biol. 66 (2004), 259-268.

[7] Hu, Teng, Jia and Zhang, Complex dynamical behaviors in a discrete eco-epidemiological model with disease in prey, Adv. Difference Equ. (2014), 2014:265.

[8] M. Koopmans, B.Wilbrink, M. Conyn, G. Natrop, H. van der Nat and H. Vennema, Transmission of H7N7 avian influenza A virus to human beings during a large outbreak in commercial poultry farms in the Netherlands, Lancet. 363 (2004), 587-593.

[9] J. R. Krebs, Optimal foraging: decision rules for predators, In: Krebs, J. R., Davies, N.B.(Eds.), Behavioural Ecology: an Evolutionary approach, First ed. Blackwell Scientific Publishers, Oxford, (1978), 23-63.

[10] Lopo F. de Jesus, C. M. Silva and Helder Vilarinho, An Eco-epidemiological model with general functional response of predator to prey, preprint.

[11] Lopo F. de Jesus, C. M. Silva and Helder Vilarinho, Periodic orbits for periodic ecoepidemiological systems with infected prey, preprint.

[12] J. Mateus, A Nonautonomous Discrete Epidemic Model with Isolation, Int. J. of Difference Equ., 11 (2016), 105-121.

[13] R. E. Mickens, Discretizations of nonlinear differential equations using explicit nonstandard methods, J. Comput. Appl. Math. 110 (1999), 181-185.

[14] A. S. Purnomo, I. Darti, A. Suryanto, Dynamics of eco-epidemiological model with harvesting, AIP Conference Proceeding 1913 (2017), 020018.

[15] C. Rebelo, A. Margheri, N. Bacaër, Persistence in seasonally forced epidemiological models, J. Math. Biol. 64 (6) (2012), 933-949.

[16] C. M. Silva, Existence of Periodic Solutions for Eco-Epidemic Model with Disease in the Prey, J. Math. Anal. Appl. 53 (2017), 383-397. 
[17] P. Van den Driessche, J. Watmough. Reproduction numbers and sub-threshould endemic equilibia for compartmental models of disease transmission, Math. Biosci. 180 (2002), 29-48.

[18] W. Wang, X.-Q. Zhao, Threshold dynamics for compartmental epidemic models in periodic environments, J. Dynam. Differential Equations 20 (3) (2008), 699-717.

[19] Xiao-Qiang Zhao, Dynamical Systems in Population Biology, CMS Books in Mathematics/Ouvrages de Mathématiques de la SMC, 16 (2003), Springer-Verlag, New York.

[20] Xingge Niu, Tailei Zhang, Zhidong Teng, The asymptotic behavior of a nonautonomous ecoepidemic model with disease in the prey, Applied Mathematical Modelling 35 (2011), 457-470.

[21] Yang Lu, Xia Wang, Shengqiang Liu, A non-autonomous predator-prey model with infected prey, Discrete Contin. Dyn. Syst. B 23 (2018), 3817-3836.

L. F. de Jesus Departamento de Matemática, Universidade da Beira Interior, 6201-001 Covilhã, Portugal

E-mail address: lopo.jesus@ubi.pt

C. M. Silva, Departamento de Matemática, Universidade da Beira Interior, 6201-001 Covilhã, Portugal

E-mail address: csilva@ubi.pt

H. Vilarinho, Departamento de Matemática, Universidade da Beira Interior, 6201001 Covilhã, Portugal

E-mail address: helder@ubi.pt 